\title{
Digitale Lösungssammlung von Konstruktionsprinzipien für die Agile Entwicklung von Leichtbaustrukturen für Luftfahrzeuge
}

Jutta Abulawi, Maximilian Weigand

Dieser Beitrag beschreibt ein einfaches Vorgehen für das Sammeln, Dokumentieren und Strukturieren von unstrukturiertem, teilweise nur implizit vorhandenem Wissen über mögliche Konstruktionslösungen aus einem speziellen Anwendungsbereich. Dabei wird ein agiler, auf Wissensgraphen basierender Ansatz verfolgt. Kurzbeschreibungen von Konstruktionsprinzipien werden als Lösungsbausteine ungeordnet in einem digitalen Lösungsspeicher abgelegt und dann über Beziehungen miteinander verknüpft. Ergänzend werden Beziehungen der Bausteine zu Eigenschaften, Kategorien und weiteren Objekten definiert. Die Lösungsbausteine können in Umfang und Detaillierungsgrad variieren. Das Vorgehen unterscheidet sich vom tabellarischen Aufbau eines Konstruktionskatalogs, bei dem das zu dokumentierende Wissensgebiet komplett erschlossen und gemäß einem festen Ordnungsschema gegliedert sein muss. Die praktische Umsetzung des hier vorgestellten Vorgehens erfolgte in der Vorentwicklung für die Flugzeugstrukturkonstruktion, wo kontinuierlich neue Konstruktionslösungen erfunden und weiterentwickelt werden und eine digitale, einfach erstellbare und leicht erweiterbare Lösungssammlung als Nachschlagewerk und Inspirationsquelle benötigt wurde.

Keywords: Konstruktionsprinzip, digitale Lösungssammlung, Wissensgraph, Flugzeugbau

\section{Einleitung}

Gerade in der kreativen Phase der Produktentwicklung ist Inspiration durch die Analyse zahlreicher vorhandener Lösungsprinzipien und Erfindungen wichtig, um neue Ideen zu entwickeln. Konstrukteure mit wenig Erfahrung in einem speziellen Aufgabenbereich, z.B. Berufsanfänger oder Konstrukteure in Arbeitnehmerüberlassung, stehen vor dem Problem, dass das Wissen über bereits erprobte oder zumindest erfundene Lösungsprinzipien in den Konstruktionsabteilungen nicht zentral dokumentiert wird und auch nicht in Lehrbüchern oder verfügbaren Konstruktionskatalogen zu finden ist. Stattdessen ist es in verschiedensten Dokumenten auf unterschiedlichste Weise repräsentiert oder existiert nur in den Köpfen von Experten. 
Ein Beispiel für ein solches Aufgabengebiet ist die Konstruktion von Bauteilen und Baugruppen für die Primär- und Sekundärstruktur von Großraumpassagierflugzeugen. Die Konsultation von Bauunterlagen bereits hergestellter Flugzeuge führt nicht zu neuartigen Konstruktionslösungen, und es gibt keine passenden Konstruktionskataloge. Einige Konstruktionsideen aus früheren Projekten sind in Patenten dokumentiert, andere sind in Skizzen, 3D-CAD-Modellen oder physischen Funktionsmustern umgesetzt. Wer in einem solchen Aufgabengebiet neu und innovativ tätig werden soll, bekommt schwer einen Überblick über den Lösungsraum und kann die Erkenntnisse früherer Projekte kaum als Inspirationsquelle für eigene Entwicklungen nutzen.

\section{Stand der Technik}

Um Spezialwissen in der Konstruktion verfügbar zu machen, muss es gesammelt, aufbereitet und in geeigneten Repräsentationsformen zugänglich gemacht werden. Neben den traditionellen Ansätzen der Wissenssammlung in Normen, gedruckten Konstruktionsrichtlinien und Katalogen gibt es mit fortschreitender Verfügbarkeit digitaler Autorensysteme den Wunsch nach Speicherung von Konstruktionswissen in digitaler Form.

\section{Konstruktionskataloge für das methodische Konstruieren}

Bei der Bearbeitung einer Konstruktionsaufgabe hängt die Güte des Endergebnisses stark von der erfolgreichen Suche nach geeigneten Lösungen ab. Die Lösungsfindung kann durch Wissenssammlungen unterstützt werden, die bewährte Lösungsprinzipien in meist zweidimensionalen Ordnungsschemata präsentieren (Pahl, Beitz, Feldhusen \& Grote: 2007, 145). Erfüllt eine solche Lösungssammlung spezielle Anforderungen bezüglich ihres Inhalts, ihres Aufbaus und ihrer Vollständigkeit, wird sie als Konstruktionskatalog bezeichnet (Roth: 2001, 1). Für das methodische Konstruieren von Maschinen und Apparaten sind Konstruktionskataloge mit spezieller inhaltlicher Aufbereitung seit vielen Jahrzehnten im Einsatz (Koller \& Kastrup: 1998, 27). Auch das als TRIZ bekannte erfinderische Problemlösen nutzt Kataloge, um speziell aufbereitete Lösungsmuster systematisch auffindbar zu präsentieren (Orloff: 2006, 137).

Die Publikation von Konstruktionskatalogen in Buchform wie in (Roth: 2001) verleiht ihnen den statischen, analogen Charakter eines gedruckten Nachschlagewerks und passt zum hohen Anspruch an Inhalt und Vollständigkeit und dem tabellarischem Aufbau. Obwohl Lösungssammlungen als unverbindliche Zusammenstellung gelten (Roth: 2001, 2), benötigen sie auch ein systematisches Zusammenstellungsprinzip und einheitliche Aufbereitungs- und Darstellungsformen. Die hierarchische Gliederung der 
Lösungselemente erfordert eine gute Taxonomie-Definition zu Beginn der Katalogisierung. Dieser erste Schritt setzt die Bekanntheit des abzubildenden Wissensgebiets voraus. Ist das zu speichernde Wissen nicht vollständig bekannt, weil es begleitend zu seiner Exploration dokumentiert und genutzt werden soll, dann erscheinen agile, digitale Ansätze zur Wissensspeicherung praktikabler als starre Tabellen mit vordefinierten Ordnungsschemata.

\section{Digitale Speicherung von vernetzten Informationen}

Miteinander zusammenhängende Daten lassen sich in Form von Graphen repräsentieren. Knoten stellen darin einzelne Einheiten dar, während Kanten die Beziehungen zwischen den Einheiten abbilden (Robinson, Webber \& Eifrem: 2015, 1). Diese Art von Daten kann in Graphendatenbanken gespeichert werden, die vor allem zur Verarbeitung von stark vernetzten Daten verwendet werden (Pokorný: 2015, 59).

Ein extrem großes, dynamisches Netzwerk mit vielfach vernetzten Daten ist das World Wide Webs (WWW). Zur Beschreibung der darin verfügbaren Ressourcen (z. B. Webseiten, Dokumente, Videos) und zur rechnerinternen Verarbeitung dieser Beschreibungen wurde das Resource Description Framework (RDF) entwickelt. Mit dem Prinzip des RDF lassen sich Informationen über jegliche Arten von Ressourcen in maschinenlesbarer, strukturierter Form repräsentieren. Die Informationen werden als Wort-Tripel in der Form <subject $><$ predicate $><$ object $>$ ausgedrückt. Dabei wird ein Tripel als statement bezeichnet. Das Subjekt ist im RDF die Bezeichnung der zu beschreibenden resource, z.B. eine Website oder ein Dokument. Das Prädikat heißt im RDF property und besteht aus einem Verb und den für die Satzbildung nötigen Präpositionen, Artikeln etc. Im statement erklärt das Prädikat die Beziehung zwischen dem Subjekt und dem Objekt, das zum Beispiel der Name einer weiteren Ressource sein kann. (Schreiber \& Raimond: 2014)

Mit einer Ontologie wird das Meta-Modell eines Graphen definiert, d.h. dass grundlegende Begriffe und Beziehungen und deren Kombinationsregeln festgelegt werden (Neches et al.: 1991, 40). Ein Beispiel ist die Definition der Klassen „Buch“, "Autor" und "Leser". Die Klassendefinitionen legen fest, welche Beziehungen untereinander möglich sind, z.B. ergibt "geschriebenVon" nur Sinn als Beziehung zwischen Buch und Autor, nicht jedoch zwischen Buch und Leser. Das Anlegen falscher oder widersprüchlicher Beziehungen kann in geeigneten Softwareumgebungen verhindert oder durch nachträgliche Konsistenzprüfungen erkannt werden. Diese Überprüfung ist Teil des sogenannten „Reasonings“ (Dengel: 2012, 174). 
Ein verbreitetes Werkzeug zum Aufbau von Ontologien ist die Web Ontology Language (OWL) (W3C OWL Working Group: 2012). Ontologien werden in vielen Fachdisziplinen zur Wissensmodellierung genutzt, z.B. in der Biologie (Ashburner et al.: 2000, 25). Auch im Ingenieurswesen werden Ontologien verwendet, z.B. für die funktionsorientierte Beschreibung von technischen Systemen (Gaag: 2010, 15).

\section{Wikis als interaktive digitale Lexika}

Wikis gehören zu den am häufigsten genutzten Social Software-Anwendungen für den Austausch von Informationen unter den Benutzern (North: 2016, 290). Zur Unterstützung des Wissensmanagements nutzen viele Unternehmen interne Wikis, in denen Mitarbeiter ihr Wissen über Prozesse, Produkte usw. zentral dokumentieren und anderen Angehörigen des Unternehmens zur Verfügung stellen können (Ebersbach, Krimmel, \& Warta: 2008, 133 \& 138). Eine Besonderheit von Wikis ist die Möglichkeit des gemeinsamen Erstellens und Bearbeitens von Inhalten, d.h. Benutzer besitzen weitgehende Lese- und Schreibrechte. Das größte Wiki ist die im Internet Online-Enzyklopädie Wikipedia.

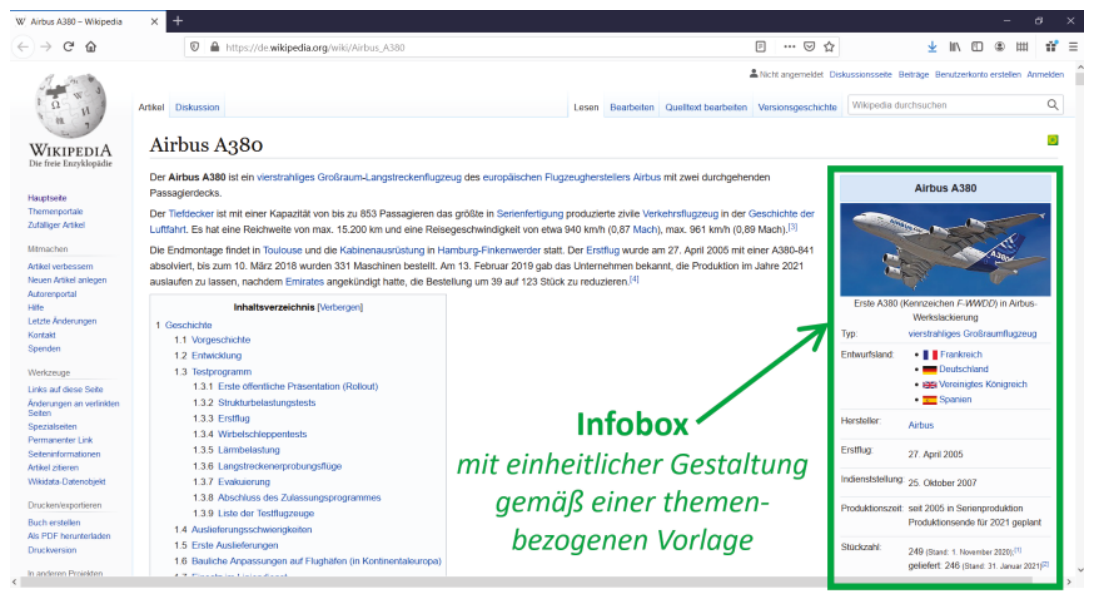

Abbildung 1: Beispiel einer Wikipedia-Seite mit Infobox

Jede Wikipedia-Seite enthält unter ihrer Überschrift einen Artikel, der Fließtext, Tabellen, Abbildungen, eingebettete Filme usw. enthalten kann. Der Aufbau von WikipediaSeiten diente als Inspiration für die Gestaltung der hier vorgestellten Lösungssammlung. Am rechten oberen Rand des Wikipedia-Artikels in Abbildung 1 erscheint eine Infobox mit wichtigen Informationen zu dem im Artikel beschriebenen Gegenstand. In Wikipedia sorgen themenbezogene Infobox-Vorlagen dafür, dass die für das jeweilige 
Thema relevanten Angaben in standardisierter Form angezeigt werden (Lange, Böhm \& Neumann: 2010, 5). Die textuellen Einträge der Infobox haben die Form <Attribut $><$ Wert $>$.

\section{Digitale Notizbücher als Vorstufe zu Wikis}

Als Vorstufe zu einem Wiki kann ein digitales Notizbuch für die Wissenssammlung in einem abgegrenzten Themengebiet verwendet werden. Digitale Notizbuch-Software ist vorrangig für das schnelle Notieren von Informationen gedacht. Für Vorlesungsmitschriften und die Prüfungsvorbereitung nutzen Ingenieurstudierende häufig digitale Notizbücher und sind deshalb mit ihrer Anwendung gut vertraut. Auch in Unternehmen werden digitale Notizbücher als einfach handhabbare Hilfsmittel für das Wissensmanagement eingesetzt. Beispielhaft berichtet North über die Sicherung von Erfahrungswissen über Produkte und Prozesse bei einem Automobilhersteller und die Dokumentation guter Konstruktionslösungen und häufiger Konstruktionsfehler in einem Ingenieurbüro mit Lotus Notes (North: 2016, 152 \& 9). Für die hier vorgestellte Lösungssammlung wurde die Software Microsoft OneNote verwendet, weil sie bekannt und verfügbar war. Mit OneNote lassen sich digitale Notizbücher leicht erstellen, nachträglich mit Schlagwörtern versehen und mit anderen Benutzern teilen. Dank des geringen Installations- und Einrichtungsaufwands eignet sich OneNote gut für die prototypische Umsetzung einer digitalen Lösungssammlung.

\section{Methode}

Das im Folgenden erläuterte Vorgehen zur Erstellung und Benutzung der digitalen Lösungssammlung bezieht sich auf den mit OneNote erstellten Prototypen einer digitalen Lösungssammlung für ein Spezialgebiet der Strukturkonstruktion im Flugzeugbau.

OneNote ermöglicht die einfache, multimediale Repräsentation von Wissen in einem virtuellen Notizbuch mit nahezu beliebig vielen Notizbuchseiten von praktisch unbegrenzter Seitenlänge. Auf jeder Seite können Fließtext, Tabellen, Formeln, Handskizzen und Audioaufnahmen erzeugt und Grafiken, Videos, Dokumente und Verknüpfungen zu externen Ressourcen eingefügt werden. Das Notizbuch lässt sich in Abschnitte gliedern und im Nachhinein umsortieren. Zu Abschnitten, Seiten oder einzelnen Elementen auf einer Seite lassen sich Verknüpfungen erzeugen und an beliebiger Stelle im Notizbuch einfügen. So kann die Verknüpfung zwischen Wissenselementen als Kernelement dieser Methode unkompliziert realisiert werden. Die Sinnhaftigkeit der Verknüpfungen verantwortet der Ersteller, da in OneNote kein objektorientiertes Meta- 
modell definierbar ist und OneNote keine Konformität mit einem externen Metamodell überprüfen kann. Diese Einschränkung ist für einen Prototypen annehmbar, jedoch nicht für komplexe Wissenssammlungen.

\section{Wichtige Schritte zum Erstellen des Lösungskatalogs}

Die einzelnen Schritte zum Erstellen und Benutzen der Lösungssammlung werden teilweise parallel oder in Iterationsschleifen durchlaufen. Das Vorgehensmodell in Abbildung 2 zeigt wesentliche Schritte und ausgewählte Ablaufszenarien.

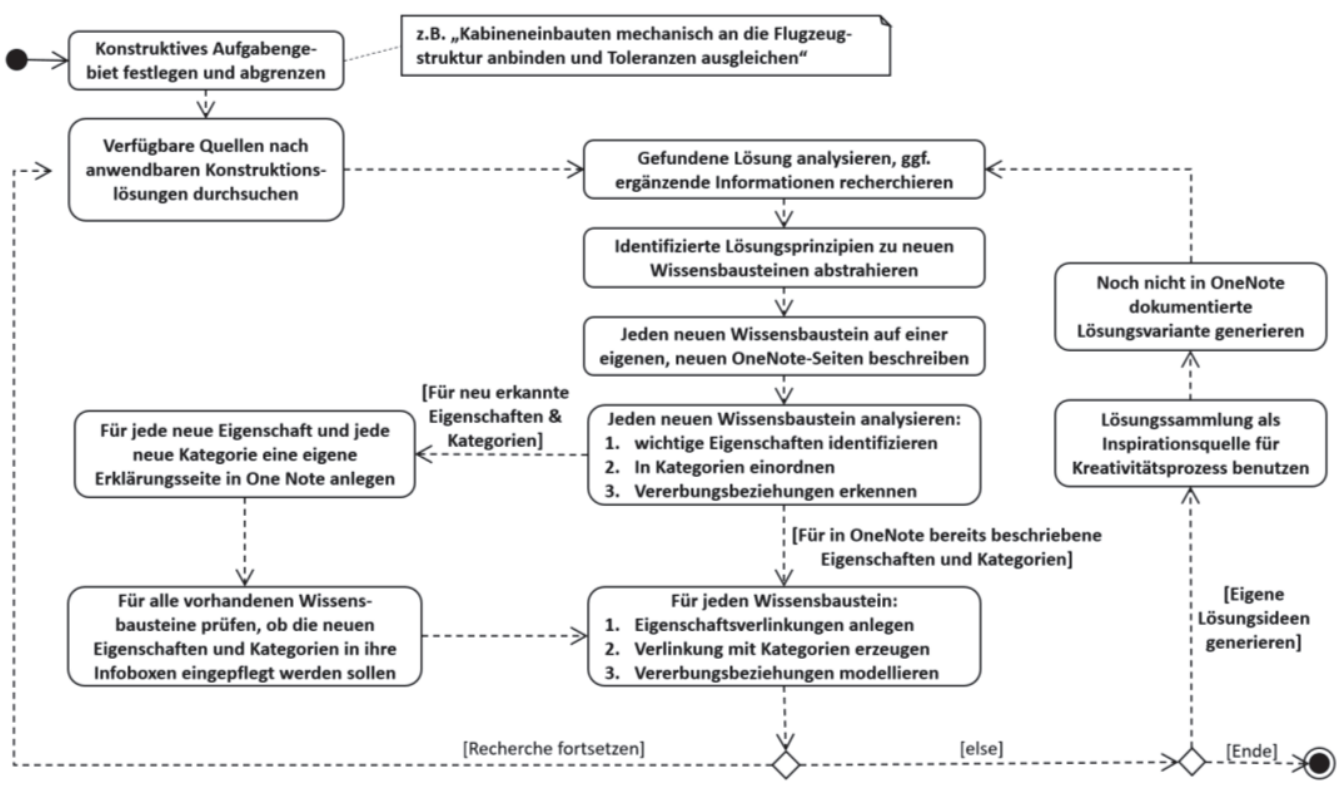

Abbildung 2: Flussdiagramm zum Vorgehen

\section{Schritt 1: Konstruktives Aufgabengebiet für die Lösungssammlung festlegen}

Zu Beginn werden die technische Domäne und die hierin durch konstruktive Lösungen zu realisierenden technischen Funktionen festgelegt. Diese Fokussierung hilft bei der Konzentration der Recherche auf relevantes Wissen. Im beschriebenen Beispiel wurden Lösungsprinzipien für Strukturanbindungen in der Flugzeugkabine mit fertigungsund montagebedingtem Toleranzausgleich zusammengetragen. 


\section{Schritt 2: Konstruktionslösungen und Lösungsprinzipien suchen}

Für das definierte konstruktive Aufgabengebiet und die konstruktiv zu realisierenden Funktionen wird in geeigneten Quellen nach bekannten Lösungen gesucht, z.B. in Patenten, bereits vorhandenen digitalen Lösungssammlungen, CAD-Modellen oder Zeichnungen. Ebenso können Experten befragt werden, damit ihr implizites Wissen über Lösungen in die Sammlung einfließt.

Die an der Recherche beteiligten Personen erhalten eine gute Übersicht über das jeweilige Wissensgebiet. Sind sie selbst Produktentwickler, können sie bei der Recherche oder auch bei der anschließenden Dokumentation zur Definition eigener, neuer Lösungsprinzipien inspiriert werden, die sie dann bestenfalls direkt in die Lösungssammlung mit einpflegen.

\section{Schritt 3: Gefundene Lösungen dokumentieren}

Die Dokumentation der Wissenselemente erfolgt durch Beschreiben jedes Lösungsprinzips auf einer separaten Notizbuchseite. Zur Beschreibung dienen kurze Texte und Abbildungen, die neu erstellt oder aus vorhandenen Dokumenten übernommen werden. Es können auch Druckansichten von Dokumenten oder die Dokumente selbst in eine OneNote-Seite eingebettet werden, dies kann aber die Übersichtlichkeit der Lösungssammlung verringern.

\section{Analyse der Lösungsbausteine und Anlegen von Verknüpfungen}

Der Kern der Methode ist nicht das Beschreiben der Lösungen, sondern das Anlegen von Verknüpfungen zwischen einzelnen Wissensbausteinen, um die Navigation in der Lösungssammlung und die gezielte Suche nach Lösungsbausteinen zu ermöglichen. Dabei wird jedes Lösungsprinzip in verschiedenen Schritten analysiert. Für die Lösungssammlung zu Strukturanbindungen in der Flugzeugkabine sind dies:

- Eigenschaften,

- Kategorien,

- Vererbungsbeziehungen.

\section{Eigenschaften}

Die einem Lösungsbaustein zugeordneten Eigenschaften können genutzt werden, um zu prüfen, ob er die Konstruktionsanforderungen erfüllt und um ihn mit anderen Lösungsbausteinen zu vergleichen. Für jede Eigenschaft wird eine Beschreibungsseite im 
Eigenschafts-Abschnitt des Notizbuchs angelegt, sobald feststeht, dass sie eine für das Anwendungsgebiet relevante Einordnung oder Suchstrategie unterstützt. In den Wissensbaustein, für den die Eigenschaft als relevant erkannt wurde, wird sie als Verknüpfung eingefügt. Außerdem wird geprüft, ob eine neu definierte Eigenschaft auch für andere, bereits dokumentierte Lösungsbausteine bedeutsam und dort ggf. nachzupflegen ist.

Die Definition von Eigenschaften erfolgt begleitend zu Recherche und Dokumentation. Dieses agile Vorgehen erlaubt den Aufbau und die Nutzung der Lösungssammlung, bevor vollständig bekannt ist, anhand welcher Aspekte das zu erschließende Wissen durchsucht werden soll.

Abbildung 3 zeigt, wie Eigenschaften werden als Verlinkungen in der Infobox auf der OneNote-Seite des Lösungsbausteins erscheinen. Die im Gegenzug auf der OneNoteSeite der Eigenschaft eingefügte Rückverlinkung zur Seite des Lösungsbausteins ist in Abbildung 4 zu sehen. Auf beiden Seiten kann die Eigenschaft durch ein Attribut oder einen Zahlenwert spezifiziert werden.

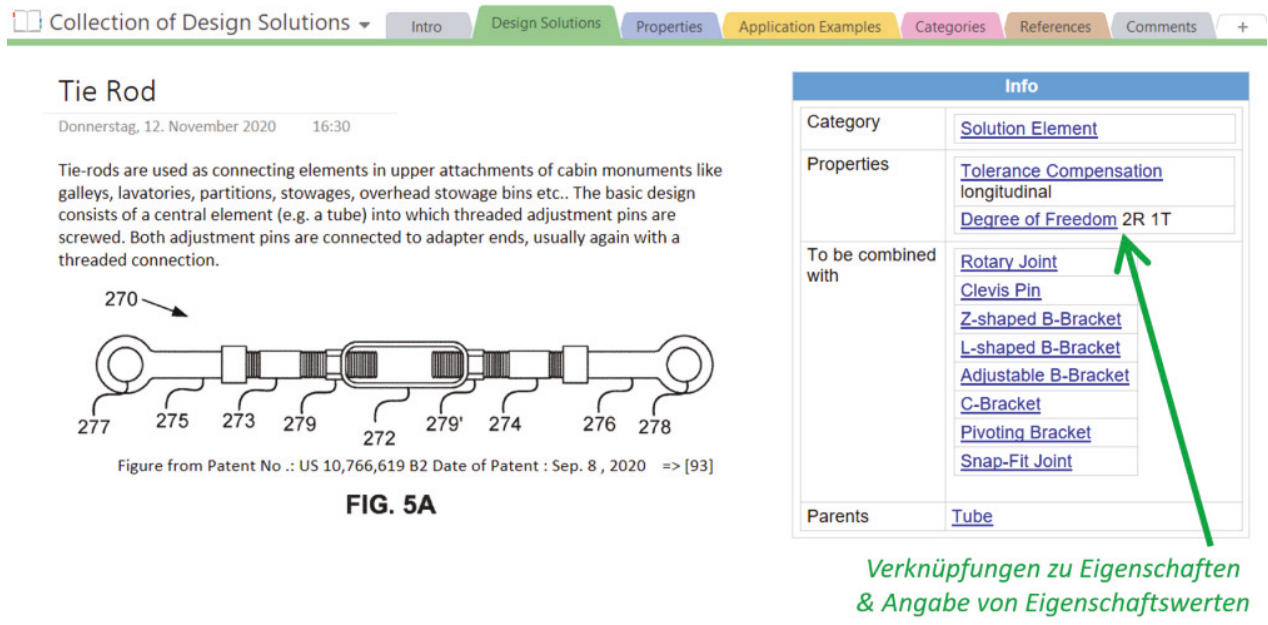

Abbildung 3: OneNote-Seite eines Lösungsbausteins mit Infobox

Die Verlinkung von der Seite des Lösungsbausteins zur Eigenschaftsseite ermöglicht das schnelle Auffinden zentral abgelegter Informationen oder Erklärungen zur Eigenschaft. Außerdem zeigen die aufgelisteten Rückverlinkungen, welche Lösungsbausteine die gleiche Eigenschaft haben, und führen den Nutzer dorthin. 


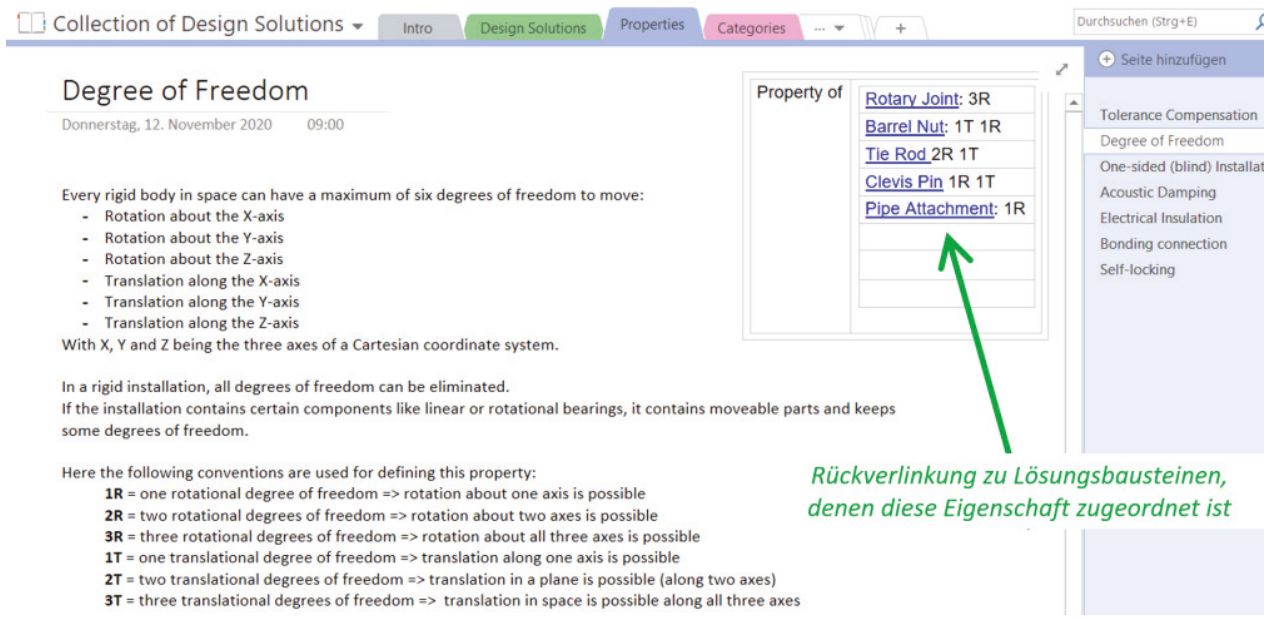

Abbildung 4: OneNote-Seite einer Eigenschaft mit Rückverlinkung zu Lösungsbausteinen

\section{Kategorien}

Das Prinzip der Verlinkung und Rückverlinkung wird analog bei der Zuordnung von Kategorien verwendet. Kategorien sind Oberbegriffe, die mehreren Lösungsbausteinen zugeordnet werden können. Jede Kategorie wird auf einer eigenen OneNote-Seite im Abschnitt „Kategorien“ erläutert. Auch Kategorien können jederzeit nachträglich definiert und in die Lösungssammlung eingepflegt werden.

Kategorien müssen sich nicht gegenseitig ausschließen. Einem Baustein können somit mehrere Kategorien zugeordnet werden. Durch dieses freie Zuordnen von Schlüsselwörtern („tagging“) entsteht eine sogenannte „Folksonomie“ (Peters: 2009, 153).

\section{Vererbungsbeziehungen und Kombinationsmöglichkeiten}

Oft basiert eine technische Lösung auf einer anderen, es besteht also ein Vererbungsverhältnis, das als Eltern-Kind-Beziehung (englisch: parent-child-relation) bezeichnet wird. Auch solche Verbindungen zwischen zwei Lösungsbausteinen können mit Verknüpfungen dokumentiert werden. Die Verlinkung führt dann zur jeweils anderen Lösung. Vor dem Link wird angegeben, in welche Richtung die Vererbungsbeziehung besteht. Abbildung 5 zeigt einen Lösungsbaustein mit Beziehungen zu einem Eltern-Element („Single Snap-Fit“) und zu einem Kind-Element („Tension Rod“). 
Durch diese wechselseitige Verlinkung entsteht eine hierarchische Ordnung. Da einem Objekt mehrere übergeordnete Objekte zugeordnet werden können, gilt sie als Polyhierarchie (Arndt: 2006, 138). Grundsätzlich kann ein Lösungsbaustein auch ohne Vererbungsbeziehungen in die Lösungssammlung aufgenommen werden. Bei Bedarf kann er später mit anderen Lösungsbausteinen in Beziehung gesetzt werden.

Die Vererbungsbeziehungen leiten den Benutzer entlang von Generalisierungs- und Spezialisierungspfaden durch die Lösungssammlung. Die Verlinkung zeigt sofort, worauf eine Lösung basiert und wie sie weiterentwickelt wurde.

Neben den Eltern-Kind-Beziehungen kann auch die Kombinierbarkeit mehrerer Lösungsbausteine zu neuen Gesamtlösungen in OneNote definiert werden. Hierzu wird in die Infobox des Lösungsbausteins die Verknüpfung zu anderen Lösungsbausteinen eingefügt, die mit ihm kombiniert werden können.

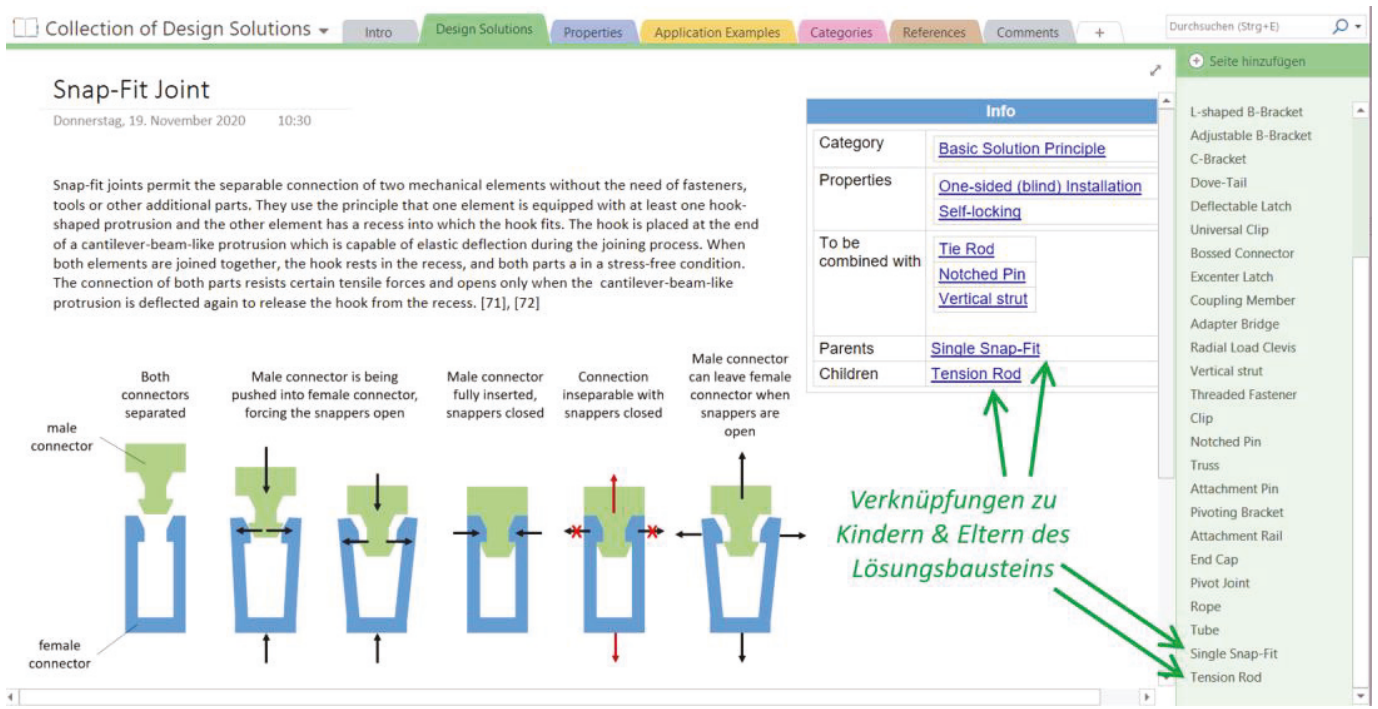

Abbildung 5: OneNote-Seite eines Lösungsbausteins mit Eltern-Kind-Beziehungen

\section{Benutzung für Dritte ermöglichen}

Um das OneNote Notizbuch Dritten zugänglich zu machen, muss es auf einem gemeinsam verwendeten Laufwerk oder in einem Cloud-Speicher wie Microsoft OneDrive gespeichert werden. In OneDrive können relativ einfach Ansichts- und Bearbeitungsrechte für verschiedene Nutzer eingeteilt werden. Das ist vorteilhaft, um die Bearbei- 
tung durch eine Gruppe von Autoren durchzuführen, die gut mit dem Metamodell vertraut ist. Das Metamodell muss dafür gut dokumentiert werden, denn die Software OneNote kann keine Verstöße gegen das Metamodell erkennen. Aber auch Betrachter sollten den grundlegenden Aufbau der Wissensdarstellung zu kennen. Für diese Benutzergruppe sollte eine Kurzanleitung erstellt werden, idealerweise direkt im Notizbuch.

\section{Benutzung, Pflege und Erweiterung}

Die vorgestellte Methode eignet sich vor allem zur Sammlung von nicht abschließend dokumentiertem und gerade entstehendem Wissen über Konstruktionen. Daher ist es möglich, die Benutzung schon bei einem insgesamt geringen Umfang der Sammlung zu starten. Die Aktualisierung des abgelegten Wissens, dessen Korrektur, sowie das Einpflegen von neuem Wissen können parallel zur Benutzung ablaufen.

Die Erweiterung und Bearbeitung des bestehenden Metamodells ist jederzeit möglich, aber mit deutlich mehr Aufwand verbunden. Sollen neue Attribute und Beziehungen eingefügt werden, müssen alle bestehenden Lösungen auf ihre Anwendbarkeit überprüft werden. Eine Bearbeitung eines Aspekts erfordert eine Überprüfung der Bedeutung aller Verbindungen, die über diesen hergestellt wurden.

\section{Bewertung}

Ein Vorteil der agilen Lösungssammlung in OneNote ist, dass eine sehr heterogene Menge an Lösungen schnell geordnet werden kann. Auch unvollständig oder skizzenhaft beschriebene Ideen lassen sich in die Lösungssammlung integrieren. Das liegt daran, dass Lösungsbausteine über die ihnen zugeordneten Eigenschaften, Vererbungsbeziehungen und Kategorien in einen Kontext gebracht werden können.

Nachteilig ist, dass ein gutes Verständnis der Methode bzw. des Meta-Modells notwendig ist, um aktiv an der Lösungssammlung mitzuwirken. Werden Inhalte planlos eingefügt oder bearbeitet, entsteht Unordnung. Um zu vermeiden, dass unwissende Bearbeiter die Lösungssammlung durcheinanderbringen, wird eine Softwareumgebung benötigt, in der das Meta-Modell für das Wissensgebiet mit Ontologien definiert und zur Erzeugung von Vorlagen und zur Konsistenzprüfung der Wissenseinträge genutzt werden kann. 


\section{Ausblick: Überführung in eine weiterentwickeltere Umgebung}

Die hier beschriebene Methode wurde prototypisch in OneNote umgesetzt. Als Anwendungsfall wurden Konstruktionslösungen dokumentiert, die in einer agilen Arbeitsgruppe entwickelt wurden. Der Umfang des zu speichernden Wissens war so begrenzt, dass die Umsetzung mit OneNote durchführbar war.

Sollen mit dieser Methode größere Wissensbestände gesammelt werden, oder gibt es stärkere formale Anforderungen, dann ist diese Umsetzung in OneNote nicht sinnvoll. Dafür wird eine Softwareumgebung mit erweitertem Funktionsumfang benötigt. Im Folgenden werden diese Funktionen betrachtet, sowie ein Vorschlag für eine Umsetzung präsentiert.

\section{Überführung in eine weiterentwickelte Umgebung}

In OneNote ist das Erstellen einer Seite zur Beschreibung eines Lösungsprinzips sehr unkompliziert. Das Format ist frei wählbar, und eine Vielzahl von Medien kann eingebunden werden. Die Verknüpfungen werden einfach mit internen Links erzeugt. Jedoch können keine Mechanismen zur Kontrolle der Verlinkungsstruktur definiert werden, die Nutzer auf Abweichungen vom Metamodell hinweisen.

Bei einem umfangreicheren Wissensdarstellungsprojekt kann dies jedoch notwendig sein. Hierfür muss das Wissensgebiet formal in einer Ontologie beschrieben werden. In einer Ontologie kann ein festes Metamodell definiert werden, dem die Struktur der Verlinkungen folgen muss. Fehler können durch sogenanntes "Reasoning" aufgedeckt werden.

Eine Ontologie ist jedoch nur für die Abbildung der Verbindungen und der Zuordnung von Eigenschaften usw. geeignet. Die einzelnen Detailbeschreibungen der Designlösungen mit Text, Abbildungen und anderen Medien können nicht in einer Ontologie abgebildet werden. Neben der Ontologie, dem Back-End, müsste ein Front-End aufgebaut werden, das dem Benutzer die Detailinformationen darstellen kann. Die Verlinkungen können dann allerdings automatisch aus dem Wissensmodell im Back-End generiert werden. Das Front-End könnte weiterhin ermöglichen, Inhalte zu erstellen und zu bearbeiten.

\section{Rollenverteilung}

Die hier beschriebene Wissenssammlung wurde von einer Person im Rahmen einer Masterarbeit erstellt. Bereits im Entstehen wurde das OneNote-Notizbuch für Betrachter freigegeben. Theoretisch wäre die Bearbeitung durch mehrere Personen möglich gewesen, fand aber aus Zeitgründen nicht statt. Diese kollaborative Bearbeitung in 
OneNote erfordert gute Absprachen der Bearbeiter, damit alle dasselbe Meta-Modell für die Verknüpfungen verwenden. Trotz bestmöglicher Kommunikation können formale Fehler auftreten, die wegen fehlender Kontrollmechanismen in OneNote nicht automatisch entdeckt werden.

In einer weiterentwickelten Umgebung ist die kollaborative Bearbeitung deutlich einfacher zu organisieren. Formale Fehler beim Erstellen und Bearbeiten von Verlinkungen können durch Kontrollmechanismen verhindert werden. Außerdem sind Benutzergruppen mit verschiedenen Berechtigungen definierbar:

- Betrachter: Nur Leserechte für Wissensbausteine,

- Bearbeiter: Lese- und Schreibrechte für Wissensbausteine,

- Administratoren: Lese- und Schreibrechte für Wissensbausteine und Meta-Modell.

\section{Webanwendung}

Die benutzerfreundlichste Umsetzung wäre die Entwicklung einer eigenständigen Webanwendung, mit der die Lösungssammlung erstellt, bearbeitet und betrachtet werden kann. Der Aufwand hierfür ist jedoch hoch und erfordert kompetente Softwareentwickler. Die prototypische Umsetzung im digitalen Notizbuch kann auch für die professionelle Entwicklung einer Webanwendung wichtige Erkenntnisse liefern und ist als Pilotprojekt empfehlenswert.

\section{Literaturverzeichnis}

Arndt, H. (2006): Integrierte Informationsarchitektur. Die erfolgreiche Konzeption professioneller Websites. Berlin, Heidelberg: Springer-Verlag.

Ashburner, M., Ball, C. A., Blake, J. A., Botstein, D., Butler, H., Cherry, J. M., Davis, A. P., Dolinski, K., Dwight, S. S., Eppig, J. T., Harris, M. A., Hill, D. P., Issel-Tarver, L., Kasarskis, A., Lewis, S., Matese, J. C., Richardson, J. E., Ringwald, M., Rubin, G. M., \& Sherlock, G. (2000): Gene ontology: tool for the unification of biology. The Gene Ontology Consortium. In: Nature genetics, 25 (1), 25-29.

Dengel, A. (Hrsg.) (2012): Semantische Technologien. Grundlagen - Konzepte - Anwendungen. Heidelberg: Spektrum Akademischer Verlag.

Ebersbach, T., Krimmel, K. \& Warta, A. (2008): Auswahl und Aussage von Kenngrößen innerbetrieblicher Wiki-Arbeit. In: Alpar, P. \& Blaschke, S. (Hrsg.): Web 2.0 - Eine empirische Bestandsaufnahme, 131-155,. Wiesbaden: Vieweg+Teubner.

Gaag, A. (2010): Entwicklung einer Ontologie zur funktionsorientierten Lösungssuche in der Produktentwicklung. Dissertation. München: Technische Universität München. 
Koller R., Kastrup N. (1998) Informationsspeicher für die Konstruktion. In: Prinziplösungen zur Konstruktion technischer Produkte, 27-48. Berlin, Heidelberg: Springer.

Lange, D., Böhm, Chr. \& Nauman, F. (2010): Extracting Structured Information from Wikipedia Articles to Populate Infoboxes. Universitätsverlag Potsdam

Neches, R., Fikes, R. E., Finin, T., Gruber, T., Patil, R., Senator, T. \& Swartout, W. R. (1991): Enabling Technology for Knowledge Sharing. In: Al Magazine, 12 (3), 36-56.

North. K. (2016): Wissensorientierte Unternehmensführung, Wissensmanagement gestalten. 6. Auflage. Wiesbaden: Gabler.

Orloff, M. (2006): Grundlagen der klassischen TRIZ, Ein praktisches Lehrbuch des erfinderischen Denkens für Ingenieure. 3. Aufl. Berlin, Heidelberg: Springer.

Pahl, G., Beitz, W., Feldhusen, J. \& Grote, K.-H. (2007): Pahl/Beitz Konstruktionslehre. Grundlagen erfolgreicher Produktentwicklung Methoden und Anwendung. 7. Aufl. Berlin, Heidelberg: Springer.

Peters, I. (2009): Folksonomies. Indexing and retrieval in Web 2.0. Berlin: Walter de Gruyter.

Pokorný, J. (2015): Graph Databases: Their Power and Limitations. In: Saeed K., Homenda W. (Hrsg.): Computer Information Systems and Industrial Management, 58-69, Cham: Springer.

Robinson, I., Webber, J., \& Eifrem, E. (2015). Graph databases: new opportunities for connected data. O'Reilly Media, Inc.

Roth, K. (2001): Konstruieren mit Konstruktionskatalogen, Band 2: Kataloge. 3. Aufl. Springer, Berlin, Heidelberg.

Schreiber, G. \& Raimond, Y. (2014): RDF 1.1 Primer. W3C Working Group Note 24 June 2014.

https://www.w3.org/TR/rdf11-primer/, veröffentlicht 2014, abgerufen am 22.03.2021.

W3C OWL Working Group (2012): OWL 2 Web Ontology Language. Document Overview (Second Edition). W3C Recommendation 11 December 2012. https://www.w3.org/TR/owl-overview/, veröffentlicht 2012, abgerufen am 22.03.2021.

\section{Kontakt}

Prof. Dr.-Ing. Jutta Abulawi

Hochschule für Angewandte Wissenschaften Hamburg

Fakultät Technik und Informatik

Department Fahrzeugtechnik und Flugzeugbau

Berliner Tor 9, 20099 Hamburg

jutta.abulawi@haw-hamburg.de

Maximilian Weigand, M.Sc.

Helmut-Schmidt-Universität / Universität der Bundeswehr Hamburg

Institut für Automatisierungstechnik

Holstenhofweg 85, 22043 Hamburg

maximilian.weigand@hsu-hh.de 